\title{
Influence of the Inclusion Particles Present in the Immediate Vicinity of a Crack on Stress Variation
}

\author{
V. Goanță ${ }^{1, a}$, M. Mihălcuț \\ ${ }^{1}$ Techn. University “Gh. Asachi” Iaşi, Strength Department, Bd. Mangeron, 700050, Romania
}

\begin{abstract}
Fracture toughness occurring in a material that has micro-cracks originating in its manufacture process may be improved by particle addition, these particles having different properties than the basic material [1]. This paper includes the outcome of a research on the influence of softer or harder particles added to the basic material, on the stresses occurring in the immediate vicinity of micro-cracks. Our research is designed to determine to what extent this process may result into preventing a crack from advancing into the material subject to unfavorable loads, depending on the direction of that crack. The outcome we achieved using finite element analysis supports the assumption that, by adding a certain amount of particles, which have specific characteristics, to the basic material, fracture toughness may increase significantly, [2].
\end{abstract}

\section{Introduction}

Technologically speaking, particle scattering in the basic material matrix is random. Nevertheless, different volume components of the material thus obtained have similar physical and chemical characteristics, according to the new component weighing and mixing technologies.

This paper does not tackle particle addition precisely at the top of the crack, as shown by the modelling, [3]. It is however well-known that certain material has micro-cracks that originate in the very manufacture process. When subjected to static or dynamic loads, these micro-cracks may grow, becoming macro-cracks. Due to their advance, these macro-cracks inevitably reach, at some point, the immediate vicinity of an inclusion particle, as shown by the modelling.

The goal of this research is to improve crack advance prevention characteristics of materials that have cracks originating in their manufacture process. This may be achieved by considering several different types of actions. First of all, we established a correlation between the technological process parameters and the number of micro-flaws present in the finite product material. Another consideration referred to possible toughness improvements using differing methods able to reduce material brittleness and micro-flaws. Once the micro-flaws were present, we tried to control crack advance starting from the number and size of critical flaws. Mechanisms may be developed, which are able to "strengthen" a structure in the flawed material, making it less sensitive to the microcracks that occurred in the material, Figure 1, [4].

Past research used to focus on identifying both the processes leading to the occurrence of cracks and the mechanisms triggering crack advance (those related to fracture mechanics). Material manufacture technologies have obviously progressed enormously lately. Nevertheless, the existence

\footnotetext{
a e-mail: vgoanta@tuiasi.ro
}

This is an Open Access article distributed under the terms of the Creative Commons Attribution-Noncommercial License 3.0, which permits unrestricted use, distribution, and reproduction in any noncommercial medium, provided the original work is properly cited. 
of manufacture micro-cracks in these materials is unquestionable. The question that should be answered is whether the existence of these micro-cracks may be tolerated without jeopardizing the structural integrity of the material during operation [5].

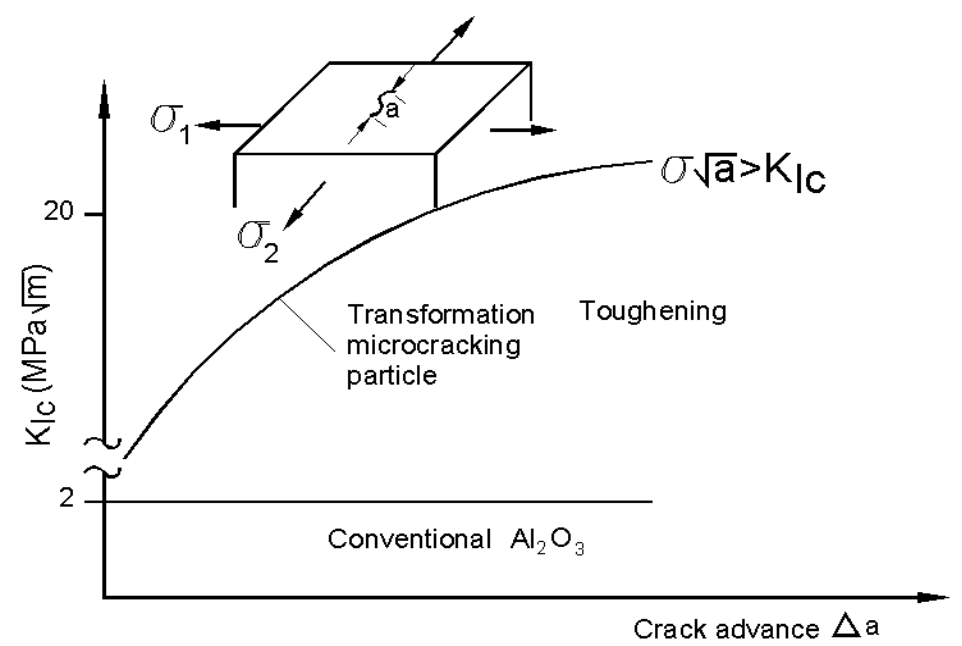

Fig. 1. Resistance curve behavior characteristically encountered in tough material.

Brittle solid resistance to crack advance may be strongly influenced by the solids' microstructure and by the addition to the microstructure of various reinforcing elements, [6]. The object of this research is the determination of the influence of inclusion particles on fracture toughness by the stresses developed at the top of the crack.

\section{Modelling through the insertion of inclusion particles}

Particles having different properties than the basic material may be scattered in the matrix of a material containing micro-cracks. This chapter describes the variation of the stresses occurring in the immediate vicinity of a micro-crack, depending on the existence, in the latter's proximity, of spherelike inclusion particles having different features. The goal of this research was the development of a method able to prevent a crack in the material subject to unfavorable loads from spreading, depending on the direction of this crack, [7]. We employed finite element analysis (ALGORmultiphysics 2009) to model a central crack affecting a plate subjected to uniaxial load, which is much bigger than the crack. Considering the geometric and load symmetry, analyzing only $1 / 8$ of the plate, applying adequate boundary conditions, should be enough, the crack being modeled in the central area in the absence of those conditions, Figure 2. Thus, the analysis may be considered as being performed on a plate having a 2 a long crack with two sphere-like inclusion particles at its ends.

The digitization network was designed to allow an easy shifting of the sphere-like particle along the $\mathrm{Y}$ axis, as shown in Figure 2a. This shifting will allow the stress variation study at the top of the crank, while the inclusion particle is moving closer to or away from the top of the crack. The uniaxial load acting on the plate according to the $\mathrm{Z}$ direction is $100 \mathrm{MPa}$, Figure $2 \mathrm{~b}$. Given the lack of boundary conditions in the central area, the nodes in this area shift modelling a crack.

Figure $2 \mathrm{~b}$ shows the boundary conditions:

- in the $\mathrm{YOZ}$ symmetry plane, $\mathrm{X}$ axis shifting and $\mathrm{Y}$ and $\mathrm{Z}$ axes rotation were prevented;

- in the YOX symmetry plane, $\mathrm{Z}$ axis shifting and $\mathrm{Y}$ and $\mathrm{X}$ axes rotation were prevented;

- in the XOZ symmetry plane, $\mathrm{Y}$ axis shifting and $\mathrm{X}$ and $\mathrm{Z}$ axes rotation were prevented;

- in the crack simulation area, the network nodes had no restriction. 


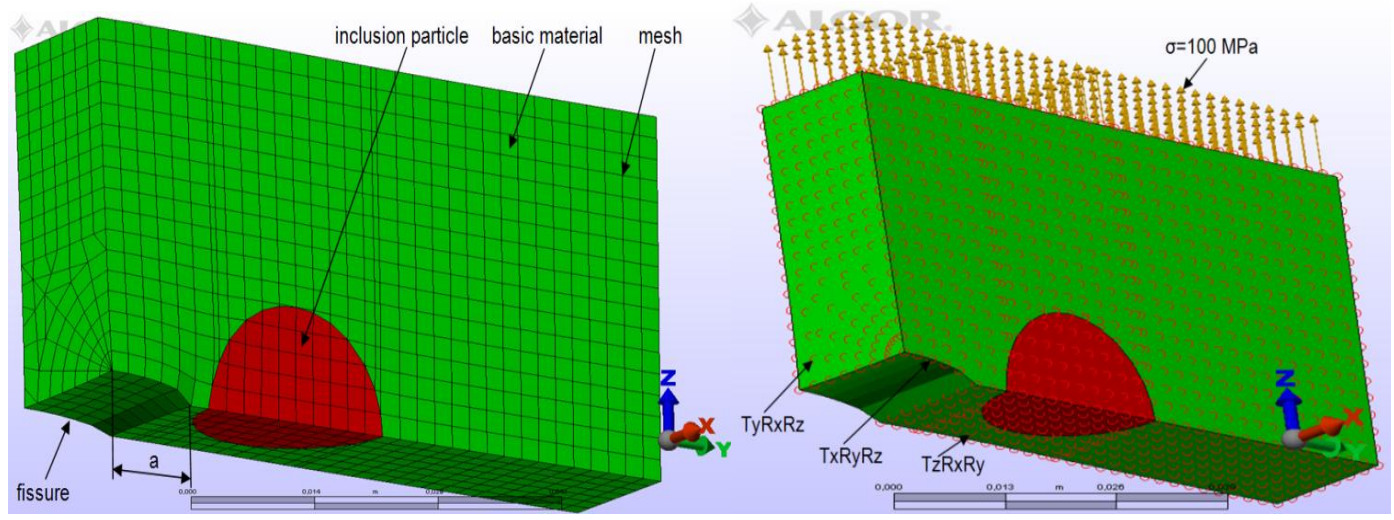

a)

b)

Fig. 2. a) Digitization network; b) Load and boundary conditions.

In order to simulate an inclusion particle, its modelling was carried out based on different material parameters for the basic material as compared to the inclusion material. We used two materials for inclusion particle simulation, whose characteristics, together of those of the basic material, are shown in Table 1.

Table 1. Characteristics of the materials used.

\begin{tabular}{|c|c|c|c|c|c|c|}
\hline Material & Simulation & $\begin{array}{c}\text { Longitudinal } \\
\text { elasticity } \\
\mathbf{m o d}\left[\mathbf{k g} / \mathbf{m}^{3}\right]\end{array}$ & $\begin{array}{c}\text { modulus, } \\
\mathbf{E} \cdot \mathbf{1 0}^{5}[\mathbf{M P a}]\end{array}$ & $\begin{array}{c}\text { Poisson's } \\
\text { ratio, } \\
\boldsymbol{\mu}\end{array}$ & $\begin{array}{c}\text { Tangent } \\
\text { modulus, } \\
\mathbf{E t} \cdot[\mathbf{M P a}]\end{array}$ & $\begin{array}{c}\text { Flow } \\
\text { limit, } \\
\boldsymbol{\sigma}[\mathbf{M P a}]\end{array}$ \\
\hline $\begin{array}{c}\text { AISI 1006 } \\
\text { steel }\end{array}$ & $\begin{array}{c}\text { Basic } \\
\text { material }\end{array}$ & 7872 & 2.05 & 0.29 & 226 & 285 \\
\hline $\begin{array}{c}\text { Al 1050-H14 } \\
\text { aluminum }\end{array}$ & $\begin{array}{c}\text { "Soft" } \\
\text { inclusion }\end{array}$ & 2705 & 0.69 & 0.33 & 71 & 103 \\
\hline Beryllium & $\begin{array}{c}\text { "Hard" } \\
\text { inclusion }\end{array}$ & 1844 & 3.03 & 0.07 & 4450 & 240 \\
\hline
\end{tabular}

\section{Elastic-plastic modelling}

The analysis was performed considering plastic modelling for von Mises materials, both on the basic material and on the inclusion particle material, since in the immediate vicinity of the top of the crack, the stress reaches values that lead to the occurrence of plastic strain in that area. The modelling was performed using nonlinear analysis of the behavior of the materials used. Thus, we employed the von Mises model to analyze material behavior after elastic limit exceeding, which triggers a uniform flow of the whole flowing area.

As concerns the elastic-plastic material behavior, the von Mises models are used when a material is subjected to loads beyond its flow limit, thus triggering the plastic strain. These models enable the user to define a certain type of strain behavior after flow occurrence in a specific area of the material. Isotropic hardening, Figure $3 \mathrm{a}$, used in this research means uniform material flow in the entire area where the flow limit was exceeded. As far as the software is concerned, a "tangent" elasticity modulus should be filled in the "strain hardening" field, which will be used when the flow limit is exceeded. 


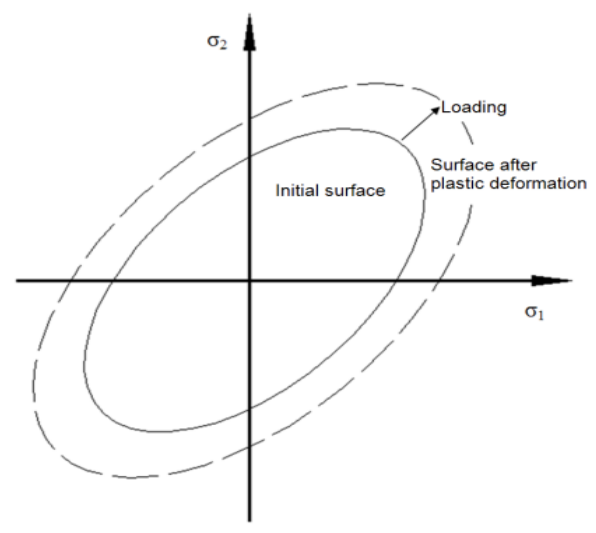

a)

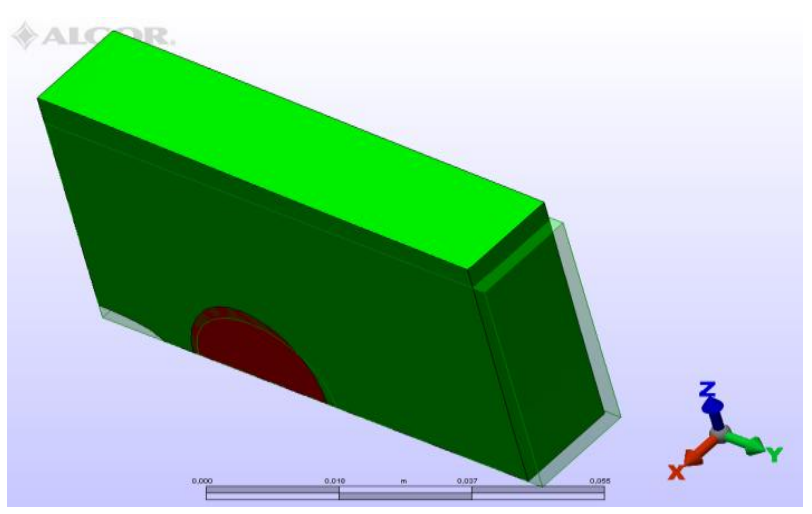

b)

Fig. 3. a) Isotropic strain; b) Model deformations.

Figure $3 \mathrm{~b}$ shows the accuracy of the crack loading, support and forming modes. As said before, due to the geometric and loading symmetry, the modelling was carried out on $1 / 8$ of the whole model. Figure $3 \mathrm{~b}$ shows the following:

- the model size increases on the direction of the load, $\mathrm{Z},-$ it becomes longer;

- the model size decreases on the other two directions, $\mathrm{X}$ and $\mathrm{Y}$;

- the three planes containing the boundary conditions, YOZ, XOZ and XOY, do not leave their initial position;

- the crack is formed in the central area, as a result of the degrees of freedom required and of the strain under constant load.

\section{Results achieved}

Using the technological process designed to produce materials containing scattered inclusion particles, the latter cannot be inserted precisely in the immediate vicinity of an existing crack. Hence, our research relied on repeated step-by-step modellings, by placing the inclusion particle in the $\mathrm{s}_{1} \div \mathrm{s}_{10}$ positions in the model's symmetry plane. Figure $4 \mathrm{a}$ shows the inclusion particle in the $\mathrm{s}_{10}$ position, and Figure $4 \mathrm{~b}$ shows the inclusion particle in the $\mathrm{s}_{1}$ point. For each $\mathrm{s}_{1} \div \mathrm{s}_{10}$ position of the soft or hard inclusion particle, the stresses on the $\mathrm{Z}$ load direction were considered, starting with node 1 .

\subsection{Soft aluminum particle}

Figures $4 \mathrm{a}$ and $4 \mathrm{~b}$ show the map of the $\sigma_{\mathrm{zz}}$ stresses, on the $\mathrm{Z}$ direction, which coincides with the load direction, if an aluminum inclusion particle that is softer than the basic material is used. We noticed a significant stress increase occurring at the top of the crack, Figure $4 \mathrm{~b}$, (point 1, step $\mathrm{s}_{1}$ ), more precisely $388 \mathrm{MPa}$ against $336 \mathrm{MPa}$ (the inclusion-free option), given the existence, in its immediate vicinity, of an inclusion particle having the properties described above. A similar stress increase at the top of the crack was also noticed when the inclusion particle was moved away from the top of the crack, see step $\mathrm{s}_{10}$, Figure $4 \mathrm{a}$. $\sigma_{\mathrm{zz}}$ stress at the top of the crack is in this case $356 \mathrm{MPa}$ against 336 $\mathrm{MPa}$ in the inclusion particle-free modelling.

Figure $4 \mathrm{~b}$ proves that there is a strong stresses concentration at the top of the crack, when the inclusion particle is in its immediate vicinity. The same figure shows that, in the inclusion particle area, the stresses are lower than in the neighboring basic material areas. We may therefore say that the stresses are not evenly distributed in the inclusion particle material. A $\sigma_{\mathrm{zz}}$ stress map discontinuity occurs at the interface between the basic material and the inclusion particle. This discontinuity is noticed in every step from $\mathrm{s}_{1}$ to $\mathrm{s}_{10}$ where the inclusion particle is modelled. 


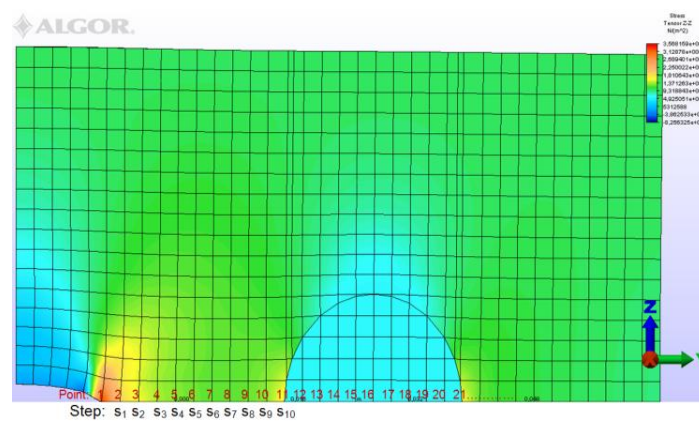

a)

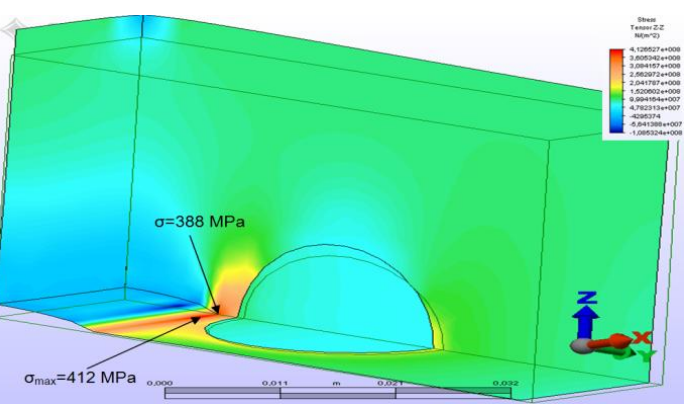

b)

Fig. 4. a) Inclusion particle displaced by the top of the crack - step $s_{10}$; b) Stresses map for the "soft" aluminum particle, on the $\mathrm{Z}$ load direction, step $s_{1}$.

Figure $4 \mathrm{~b}$ also shows that the maximum stress $\left(\sigma_{\max }=412 \mathrm{MPa}\right)$ does not occur in the model's symmetry plane, but in the immediately following node of this plane.

We traced, in Figure 5a, the $\sigma_{\mathrm{zz}}$ stress variation graphs depending on the distance from the top of the crack, for each of the $s_{1}$ through $s_{10}$ inclusion particle displacement steps, against the top of the crack. Figure $5 \mathrm{~b}$ shows in detail only the $\sigma_{\mathrm{zz}}$ stress variation graphs for the steps 1 and 10 concerning the position of the inclusion particle as compared to the top of the crack.
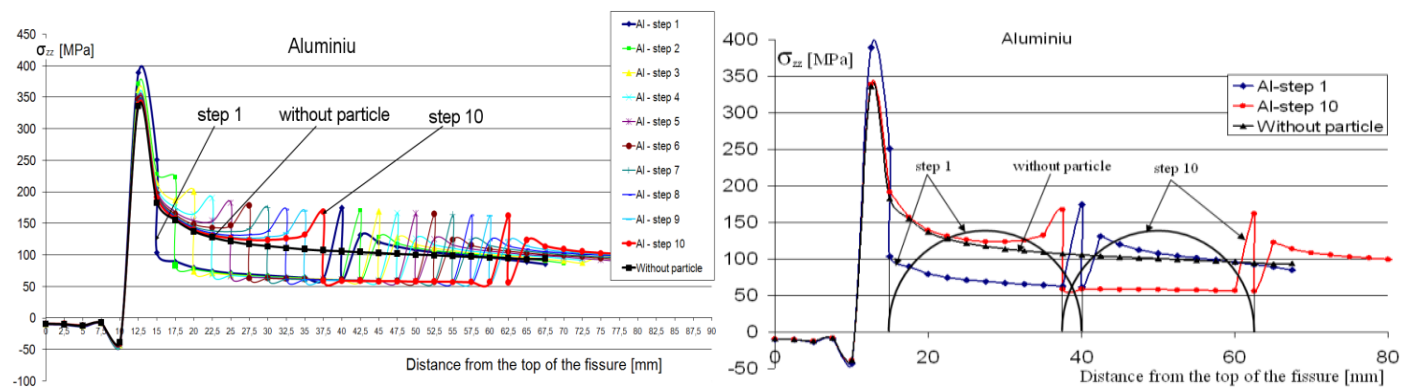

a) curves (step $1 \div$ step10) detailed view (step $1+$ step10)

Fig. 5. Stress variation on the $\left(\sigma_{\mathrm{zz}}\right)$ load direction, depending on the distance from the top of the crack, when the aluminum particle moves from step $\mathrm{s}_{1}$ to $\mathrm{s}_{10}$.

We noticed the following (see Figures $5 \mathrm{a}$ and $5 \mathrm{~b}$ ):

$>$ as compared to the inclusion-free case, the stress at the top of the crack experiences a significant increase when a softer (aluminum) inclusion particle then the basic material is inserted, and when this particle is in the immediate vicinity of the crack;

$>$ even when the inclusion particle moves towards or away from the top of the crack, the stress is higher in the network nodes closer to the top of the crack than when there is not inclusion whatsoever;

$>$ the stress in the network nodes is lower if an inclusion particle is present then if this particle is absent;

$>$ at the crack-related interface between the basic material and the particle, the $\sigma_{\mathrm{zz}}$ stress first experiences a slight increase and then a sudden drop to a much lower value than the stress value in the absence of the particle;

$>$ at the interface between the basic material and the particle opposite to the crack, the $\sigma_{\mathrm{zz}}$ stress has a marked increase followed by a sudden drop, followed again by an increase;

$>$ the stress increases again in the nodes beyond the inclusion particle, almost reaching the inclusion particle-free value. 


\subsection{Hard beryllium particle}

The second modelling consists of inserting a harder inclusion particle than the basic material that is a beryllium particle, with the mechanical and elastic characteristics shown in Table 1. Figures 6a and $6 \mathrm{~b}$ show the $\sigma_{\mathrm{zz}}$ stresses map on the $\mathrm{Z}$ direction, which coincides with the load direction.

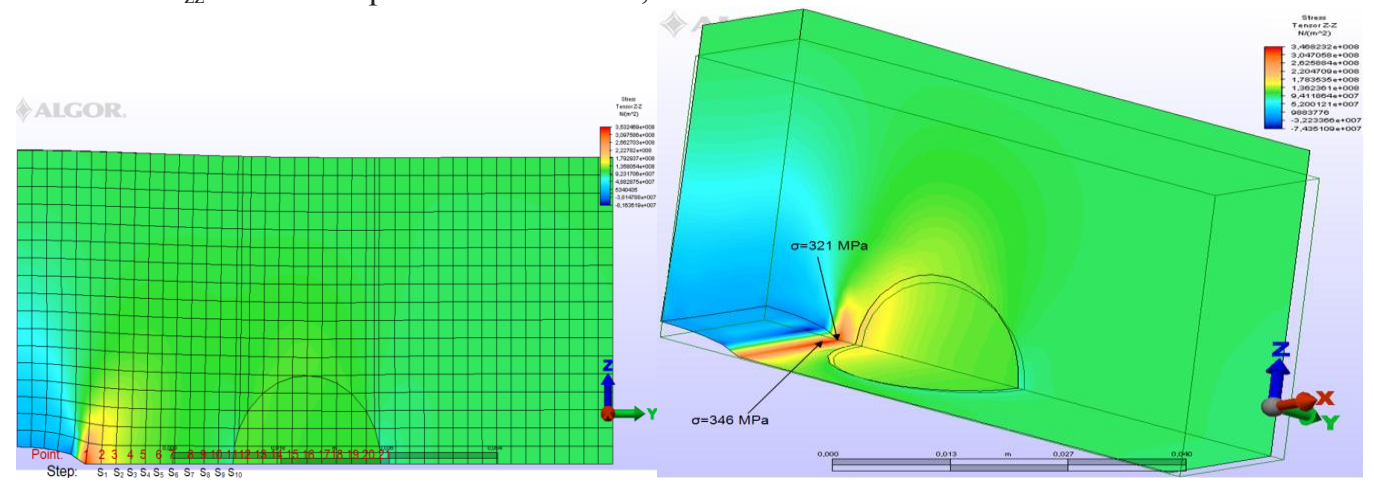

a)

b)

Fig. 6. a) Inclusion particle moving away from the top of the crack - step $s_{10}$; b) Stresses map for the "hard" beryllium particle, on the $\mathrm{Z}$ load direction, step $s_{1}$.

As compared to the inclusion-free model, we noticed a stress decrease at the top of the crack, Figure $6 \mathrm{~b}$, (point 1 , step $\mathrm{s}_{1}$ ), from $336 \mathrm{MPa}$ (the inclusion-free option) to $321 \mathrm{MPa}$, given the existence, in its immediate vicinity, of an inclusion particle having the characteristics in Table 1 . When the inclusion particle moves away from the top of the crack, the $\sigma_{\mathrm{zz}}$ stress is closer to the value occurring at the top of the crack in the inclusion-free model.

Figure 6 ( $\mathrm{a}$ and $\mathrm{b}$ ) shows that the stresses map has an almost homogeneous distribution in the inclusion particle area as well, and there are no large distortions at the interface between the matrix and particle materials. This happens both when the particle is in the immediate vicinity of the crack and when the particle is moved away from the crack. Thus, we may say that the presence of the inclusion particle harder than the basic material influences the $\sigma_{\mathrm{zz}}$ stresses map less than the presence of a softer particle than the basic material. One may notice again that the maximum stress does not occur in the model's symmetry plane, Figure 6 b.

We traced, in Figure $7 \mathrm{a}$, the variation charts of the $\sigma_{\mathrm{zz}}$ stress in relation to the distance from the top of the crack, for each step from $s_{1}$ to $s_{10}$ for the displacement of the inclusion particle in relation to the top of the crack. For details, in Figure $7 \mathrm{~b}$ we drew only the variation charts of the $\sigma_{\mathrm{zz}}$ stress, representing step 1 and step 10 concerning the arrangement of the inclusion particle in relation to the distance from the top of the crack.

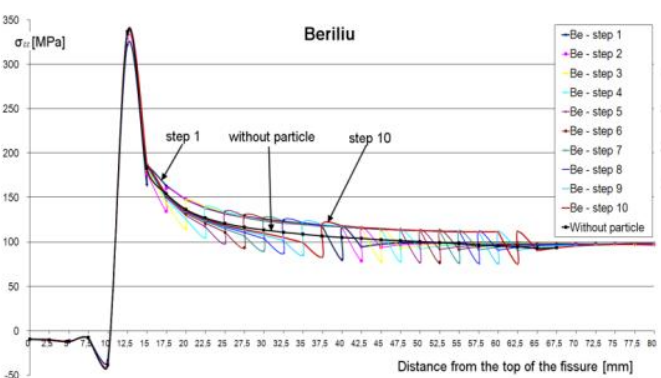

a) curves (step1 $\div$ step10)

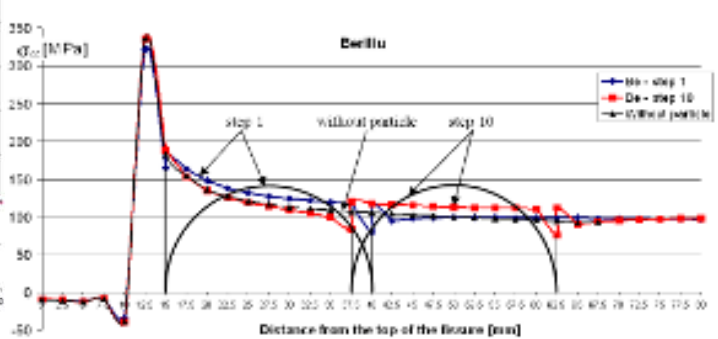

b) details (step 1+step10)

Fig. 7. The variation of the stresses in the direction of the load $\left(\sigma_{z z}\right)$ in relation to the distance from the top of the crack, for the displacement of the Beryllium particle from step $\mathrm{s}_{1}$ to step $\mathrm{s}_{10}$. 
In Figures $7 \mathrm{a}$ and $7 \mathrm{~b}$, we may notice the following:

$>$ the stress at the top of the crack presents a slight decrease when an inclusion particle "harder" than the basic material is introduced in the immediate vicinity of the crack;

$>$ regardless of the position of the particle in relation to the crack, in the nodes corresponding to the particle, the $\sigma_{\mathrm{zz}}$ stress is higher than if the particle were absent;

$>$ the stress becomes smaller again in the nodes corresponding to the material zone beyond the inclusion particle in relation to the top of the crack

$>$ at both interferences between the basic material and the particle, the $\sigma_{\mathrm{zz}}$ stress firstly presents a slight decrease (under the value of the stress if the particle were absent), and then follows a sudden increase (over the value of the stress if the particle were absent).

\section{Comparisons - Aluminum - Beryllium}

Considerations concerning the presence of the inclusion particle in the immediate vicinity of the top of the crack-step 1 .

We may notice that, through the introduction of an inclusion particle from a material that is "softer" than the basic one, the stress at the top of the crack increases in relation to the case without inclusion (388 $\mathrm{MPa}$ as opposed to $336 \mathrm{MPa}$ ), Figure 8a. Through the introduction of an inclusion particle from a material that is harder than the basic one, the stress at the top of the crack decreases insignificantly in relation to the case without inclusion (321 MPa as opposed to $336 \mathrm{MPa}$ ).

Consequently, if a micro-crack which existed in the material from the manufacturing stage advances in such a way that its top reaches the immediate vicinity of an inclusion particle whose material characteristics are of such a nature that it is "softer" than the basic material, the stress at the top of the respective crack will be higher than if the inclusion particle were absent. In these circumstances, there is an additional factor which favors the advancement of the crack. Nevertheless, if the load is applied in such a manner that the trajectory of the crack passes through the inclusion particles, due to its material characteristics and to the fact that the stress in the nodes corresponding to the inclusion particle is smaller than in the case the particle was absent, it is possible for the crack to stop in this area. If the crack "manages" to pass by the inclusion particle made from a material that is "softer" than the basic one, it will continue to advance, at fast pace, due to the fact that in the corresponding nodes after the inclusion particle the stress significantly increase again.

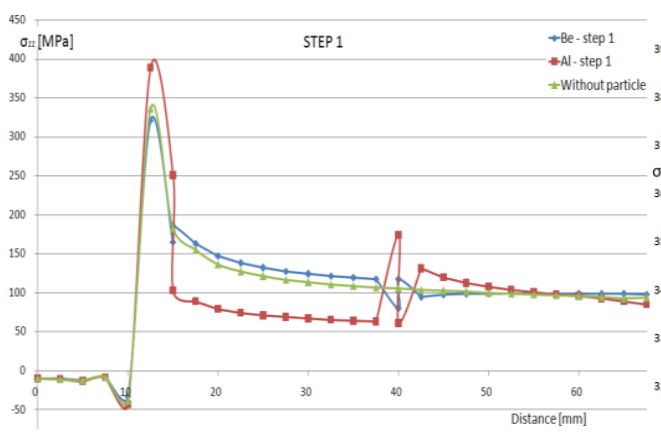

a)

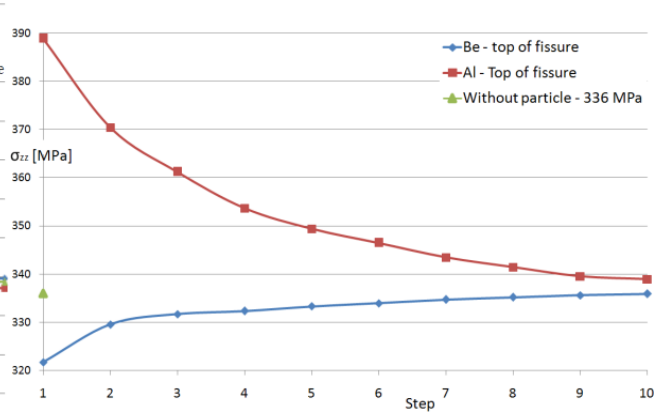

b)

Fig. 8. a) Variation of the $\sigma_{\mathrm{z}}$ stress depending on the distance in relation to the top of the crack - particle in step 1 ; b) Variation of the $\sigma_{\mathrm{z}}$ stress at the top of the crack when the particle advances from the top of the crack towards the outside.

When, through its advancement, a micro-crack that preexisted in the material from the manufacturing stage reaches the immediate vicinity of an inclusion particle whose material characteristics are of such a nature that it is "harder" than the basic material, the stress at the top of the crack will be smaller than if the inclusion particle were absent. In these circumstances, it is possible for the crack to no longer advance if we are at the limit between advancement and non- 
advancement. Nevertheless, if, under these circumstances, the crack continues to advance through the material of the particle, then it will advance at a fast pace due to the fact that the stress in the nodes corresponding to the inclusion particles is higher than the stress in the same nodes if the inclusion particle is absent. If the crack passes by the inclusion particle from a harder material than the basic one - which is very likely - it is possible for the advancement to stop because the stress in the nodes after the inclusion particle significantly decreases.

The same considerations apply when the inclusion particle is farther away from the top of the crack, with some mentions:

- for the "softer" particle, the stress at the top of the crack decreases as the inclusion particle moves further away from the top of the crack, Figure $8 b$;

- for the "harder" particle, the stress at the top of the crack increases as the inclusion particle moves further away from the top of the crack, Figure $8 \mathrm{~b}$.

\section{Conclusions}

In this chapter we performed a study concerning the variation of the stresses in the materials with cracks comprising scattered particles, with different features than those of the basic material. We noticed the fact that, via the scattering of some particles of material with different properties from those of the basic material, in the immediate vicinity of the crack, the distribution of the stresses changes.

Through the introduction of some "softer" particles than the basic material, the stress at the top of the crack significantly increases ( $388 \mathrm{MPa}$ as opposed to $336 \mathrm{MPa}$ ), and the stress in the area of the inclusion particle decreases. In these circumstances, we may perform an experimental study, from a different perspective, given the fact that, although the requirements for the advancement of a micro-crack starting from the top of the existing crack are met, it is possible for this advance to stop in the inclusion area.

Through the introduction of an inclusion particle "harder" than the basic material, the stress at the top of the crack decreases (321 MPa as opposed to $336 \mathrm{MPa}$ ). In these circumstances, although the stress in the area corresponding to the inclusion particle increases when compared to the case where the particle is absent, it is possible for the crack not to start to advance.

Acknowledgments. This work has been supported by CNCSIS-Romania under the ID_597/2007 project.

\section{References}

1. D. Bremberg, G. Dhont, Automatic crack-insertion for arbitrary crack growth, Eng. Fract. Mech. 75, p. 404-416, (2008)

2. A.P. Verma, Effective Fracture Toughness of Particulate Composites, IE(I) Journal-MM 62, 85, p. 59-64, (2004)

3. A. Ayyar, N. Chawla, Microstructure-based modelling of crack growth in particle reinforced composites, Composites Science and Technology 66, p. 1980-1994, (2006)

4. A. Bodnar, M. Chrzanowski, K. Nowak, P. Latus, Influence of small variations of initial defects upon crack paths in creeping plates - Continuum Damage Mechanics description, Eng. Fract. Mech. 75, p. 526-523, (2008)

5. T.H. Gavignan, R.A. Queeney, "Theoretical Fracture Resistance of Particle-Hardened Brittle Solids”, Fracture Mechanics. Theory and analysis, p. I.84-I.95, (1993)

6. A.J. Sánchez-Herencia, N. Hernández, R. Moreno, Fracture Behavior of Pressureless Sintered Nickel-Reinforced Alumina Composites, Key Engineering Materials Vol. 290 p. 324-327, (2005)

7. V. Goanță, V. Palihovici, "Experimental Determination of Cracking Strength", Buletinul Institutului Politehnic Iaşi, Tomul XLV(IL), fasc. 1-2, pg. 249-256, (1999) 